\title{
Effect of probiotics supplementation combined with WeChat platform health management on nutritional status, inflammatory factors, and quality of life in patients with mild-to-moderate ulcerative colitis: a randomized trial
}

\author{
Qiang $\mathrm{Ou}^{1}$, Ling Wang ${ }^{2}$, Ke Wang ${ }^{3}$, Pengtong Shao ${ }^{4}$ \\ ${ }^{1}$ Department of Anorectal Surgery, Affiliated Hospital of Jiangnan University, Wuxi, China; ${ }^{2}$ Department of Gynecology, Suzhou Hospital of \\ traditional Chinese Medicine, Suzhou, China; ${ }^{3}$ Medical department of Traditional Chinese Medicine, Wuxi Yi Bo Gang Tai Hospital, Wuxi, China; \\ ${ }^{4}$ Digestive Endoscopy Room, Wuxi Yi Bo Gang Tai Hospital, Wuxi, China \\ Contributions: (I) Conception and design: Q Ou; (II) Administrative support: L Wang; (III) Provision of study materials or patients: K Wang; (IV) \\ Collection and assembly of data: P Shao; (V) Data analysis and interpretation: All authors; (VI) Manuscript writing: All authors; (VII) Final approval \\ of manuscript: All authors. \\ Correspondence to: Qiang Ou. Department of Anorectal surgery, Affiliated Hospital of Jiangnan University, Wuxi 214122, China. \\ Email: ouqiang25@126.com.
}

Background: Ulcerative colitis (UC) is a refractory disease which is difficult to cure, is increasing in incidence, and is expensive to manage. The impact of long-term remission and recurrence in UC patients can cause a loss of confidence in patients regarding the efficacy of treatment and a lack of compliance, which creates a vicious circle. There is a lack of research on the nutritional status and quality of life (QOL) of UC patients in China. Therefore, the objective of this study was to evaluate the effects of probiotics supplementation combined with WeChat platform health management on the nutritional status, inflammatory factor levels, and QOL of patients with mild to moderate UC.

Methods: We enrolled 150 patients diagnosed with mild to moderate UC in the outpatient and inpatient department of the Affiliated Hospital of Jiangnan University between January 2018 to December 2020. Patients were randomly divided into an intervention group (probiotics supplement combined with WeChat platform) and a control group (routine follow-up). Changes to the nutritional status, inflammatory factor levels, and QOL of patients at discharge and 12 weeks after discharge were compared.

Results: After 12 weeks of intervention, the nutritional status, inflammatory factor levels, and QOL scores of the two groups were improved compared with those before 12 weeks. Nutritional status related indicators (PA, ALB, and TP) of the intervention group were significantly higher than those of the control group $(\mathrm{P}<0.05)$; the inflammatory factors (IL-6, IL-8, and TNF- $\alpha$ ) of the intervention group were significantly lower than those of the control group $(\mathrm{P}<0.05)$; the SF-36 scores (PF, RP, and $\mathrm{SF})$ in the intervention group were significantly higher than those in the control group $(\mathrm{P}<0.05)$; and the IBDQ scores $(\mathrm{BS}, \mathrm{SS}$, and $\mathrm{EF})$ in the intervention group were significantly higher than those in the control group $(\mathrm{P}<0.05)$.

Conclusions: Probiotics supplements combined with WeChat platform health management have a better curative effect on UC than conventional treatment by significantly improving the nutritional status of patients, reducing the level of inflammatory factors, and improving the QOL of patients. This intervention adds value to the management of UC patients.

Trial Registration: Chinese Clinical Trial Registry ChiCTR2 100046446.

Keywords: Ulcerative colitis (UC); WeChat; probiotics; nutritional status; quality of life (QOL)

Submitted Apr 08, 2021. Accepted for publication Jun 03, 2021.

doi: 10.21037/apm-21-1056

View this article at: http://dx.doi.org/10.21037/apm-21-1056 


\section{Introduction}

Ulcerative colitis (UC) is a chronic disease, the pathogenesis of which may be related to genetic background, environmental and luminal factors, and mucosal immune dysregulation (1). The clinical manifestations of UC are abdominal pain, diarrhea, and mucus, pus, and blood in the stool. In the past 40 years, the incidence of UC in various Asian countries has increased annually (2). The current incidence in Asia is 1.95 per 100,000 , the prevalence is 18.12 per 100,000 , and the age of onset is 20-24 years for men and 25-29 years for women (3).

$\mathrm{UC}$ has a lingering course, is difficult to cure, and attracts high medical expenses. It is recognized by the WHO as an intractable disease which seriously affects the quality of life (QOL) of patients (4). As the condition involves chronic bouts of remission and recurrence and the lives of patients are severely affected, patients are at risk of losing confidence in treatment and not complying with medical advice. At present, research on UC in China is mostly limited to the etiology, diagnosis, and treatment of the disease, and there are relatively few studies on the nutritional status and QOL of patients. Most patients with UC in China suffer a mild to moderate patients form of the disease with recurrent episodes (5), and taking into account the differences in race, economic status, cultural background, lifestyle, and medical system, relevant foreign studies cannot fully reflect the QOL of Chinese UC patients.

The WeChat platform is widely used in various fields. The medical applications of the platform have mainly involved health management issues such as disease feedback, diet intervention, and behavior education, and many researchers have used the WeChat platform to manage the health of patients to achieve positive results (6-8). However, a probiotic supplement project based on WeChat platform for patients with mild to moderate UC has not been reported nationally and internationally to date.

This aim of this study was to investigate changes in the nutritional status, inflammatory factor levels, and QOL of patients with mild to moderate $\mathrm{UC}$ receiving probiotic supplements combined with health management using the WeChat platform. The results may provide clinical data support for further improving the treatment plan of patients with mild to moderate UC in China.

We present the following article in accordance with the CONSORT reporting checklist (available at http://dx.doi. org/10.21037/apm-21-1056).

\section{Methods}

\section{Setting and study design}

We enrolled 150 patients with mild to moderate UC who were diagnosed in the Jiangnan University Affiliated Hospital between January 2018 and December 2020. Using the random number table method, patients were divided into an intervention group and control group according to the ratio of $1: 1$, and at the time of discharge, and 12 weeks after discharge, the nutritional status, inflammatory factor levels, and QOL of patients were evaluated.

The inclusion criteria were all of the following: (I) patients were 18 years old and above, normal mental and cognitive status, the could cooperate to complete the investigation and research; (II) patients met the diagnostic criteria for mild to moderate UC; (III) patients had undergone laboratory examination, ultrasound examination, gastroscopy, colonoscopy and other similar examinations such as biopsy; (IV) no complications of other important organ diseases such as those effecting the heart, liver, lung, or kidney were present; (V) patients voluntarily participated in the study and signed written informed consent. The exclusion criteria were any of the following: (I) patients who refused to participate; (II) patients who could not understand or complete the questionnaire correctly; (III) patients with severe infections and chronic wasting diseases; (IV) heart, lung, liver, or kidney dysfunction, effecting the prognosis of the disease; (V) patients with gastrointestinal tumors; (VI) patients with severe UC and Crohn's disease; (VII) pregnant or lactating women.

Both groups of patients were given conventional treatment during the hospitalization period which involved careful observation, medication, and diet and exercise guidance. After discharge from the hospital, the control group was given mesalamine (Aidisha, France Aidfa Pharmaceutical Company), 4 times/day, $1.0 \mathrm{~g} /$ time, for 12 weeks, and routine telephone follow-up. The observation group was treated with mesalazine combined with Bifidobacterium triple viable bacteria (Peifeikang, Shanghai Xinyi Pharmaceutical Co., Ltd., S10950032) three times/d, $420 \mathrm{mg} /$ time, and United States Salazine (Aidisha, France Aidfa Pharmaceutical Company), four times/d, $1.0 \mathrm{~g} /$ time, for 12 weeks, and given health management intervention on WeChat platform.

The health management team was composed of one chief physician, two attending physicians, two head nurses, 
three nurses, and one nutritionist. All team members were required to operate the WeChat application (app) proficiently, as organized by the chief physician or head nurse, and to have an appropriate level of knowledge of UC health management and health intervention. The establishment process took the following steps. (I) Mobile file establishment and real-time update: an app personal file containing demographic and other general information, diagnosis and treatment status, medication, complications, prognosis, follow-up records, and other aspects of their progress was established for the patient using the WeChat platform, and a team member was designated to carry out follow-ups every two weeks. (II) Diet survey and intervention: develop a diet plan for patients according to the existing dietary guidelines for UC (9). During the active period of UC, choose a low-fat liquid or low-fat, lowresidue semi-liquid diet, such as freshwater fish, lean meat, eggs, etc. containing high-quality protein, but avoid foods containing lactose protein, such as milk; severe patients can be given enteral nutrition preparations; Adequate intake of protein, avoid too spicy, fried foods. Observe the patient's tolerance to food and choose the right food. A daily dietary intake survey was established and explained to patients and their families during hospitalization, which included recording of the daily intake of protein, fat, carbohydrates, and energy. Targeted feedback was provided to patients on diet adjustment plans by staff monitoring the platform. (III) Health education: this took the form of video playback, animation, a publicity column, and centralized explanation, to promote UC-related knowledge and corresponding health management concepts through the WeChat platform. (IV) Answering questions and solving questions: patients were encouraged to regularly use the WeChat platform to ask questions, sort out common problems, and discuss solutions within the group. (V) Psychological intervention: attention was paid to the mental health of patients, and family members were asked to be aware of signs of suspected psychological problems. Whilst family members were encouraged to intervene and provide comfort to their loved ones during this stressful time, professional intervention was also made available. This study was conducted in accordance with the ethical principles of the Declaration of Helsinki (as revised in 2013) and has been approved by the ethics committee of the Affiliated Hospital of Jiangnan University (No. 201804367). Informed consent was taken from all the patients.

\section{Examination and determination of nutritional status}

The height and weight of patients were measured at the time of discharge and 12 weeks after discharge, and the patient's fasting venous blood was drawn in the morning at both occasions. The albumin (ALB), Total Protein (TP), and hemoglobin (HGB) were tested with an automatic biochemical analyzer (Beckman Coulter), and the level of Prealbumin (PA) was detected by enzyme-linked immunoassay. The nutritional status of the two groups, including body mass index (BMI), was also evaluated.

\section{Detection of inflammatory factors}

At the time of discharge and 12 weeks after discharge, $5 \mathrm{~mL}$ of fasting venous blood was drawn from the patient in the morning, centrifuged at 3,000 r/min for $15 \mathrm{~min}$, and the supernatant was taken and placed in a refrigerator at $-80{ }^{\circ} \mathrm{C}$ for testing. Double antibody sandwich enzymelinked immunosorbent assay (Elabscience) was then used to detect serum IL-6, IL-8, and TNF- $\alpha$ levels in accordance with the manufacturer's instructions.

\section{QOL survey}

The inflammatory bowel disease QOL (IBDQ) and 36-Item Short Form Survey (SF-36) were combined for this study. The IBDQ assesses the patient's QOL from four aspects: bowel symptoms, systemic symptoms, emotional function, and social function. There are 32 questions in total, with a total score of 32 to 224 and the higher the score, the higher the patient's QOL.

The SF-36 is divided into eight dimensions and a total of 36 items, including general health (GH, five items), physiological function (PF, 10 items), role physical (RP, four items), body pain (BP, two items), vitality (VT, four items), social function (SF, two items), role emotional (RE, three items), and mental health (MH, five items), item 2 is self-reported Changes in health do not participate in the calculation of scale scores. The score of each dimension adopts a percentile system and is calculated as follows: Each dimension score $=[$ (actual score-the lowest score of the dimension)/the difference between the highest possible score and the lowest score of the dimension] $\times 100$. The higher the score, the better QOL.

Questionnaire data collection: trained professional doctors explained the purpose and significance of the 
survey to patients who met the inclusion criteria, consent was obtained, and a face-to-face questionnaire survey was conducted. The total time to complete the questionnaire was 30-40 minutes.

\section{Statistical analysis}

Spss22.0 software was used for statistical analysis. The measurement data of normal distribution was expressed by $\bar{x}_{ \pm s}$. The paired $t$-test was used for comparison before and after treatment in the same group, and the $t$-test of independent samples was used for comparison between groups. Chi-square test was used for comparison of count data between groups and $\mathrm{P}<0.05$ was considered statistically significant.

\section{Results}

\section{Baseline data}

Of the $150 \mathrm{UC}$ patients enrolled in this study, one refused to participate and two did not meet the inclusion criteria. The remaining 147 patients were randomly divided into a control group $(n=73)$ and intervention group $(n=74)$. During the study, one patient in the control group was lost, aw were four in the intervention group. The average age of the control group was $39.07 \pm 7.67$ and there were 40 males and 33 females. The maximum level of education listed was primary school in 20 patients, secondary and higher vocational education in 15 , and junior college or above, in 38. There were 22 unmarried/divorced/widowed patients and 51 married patients. The monthly per capita income was $<2,000$ in 22 patients, 2,000-3,499 in 21 patients, $3,500-4,999$ in 17 , and $\geq 5,000$ in 13. Mild UC was diagnosed in 26 patients and moderate UC in 47 . In the intervention group the average age of the intervention group was $41.77 \pm 8.82$ and there were 40 males and 29 females. The maximum level of education listed was primary school in 15 patients, secondary and higher vocational education in 14 , and junior college or above in 40. There were $21 \mathrm{Unmarried/divorced/widowed} \mathrm{patients,}$ and 48 married patients. The monthly per capita income in this group was $<2,000$ in 19 patients, 2,000-3,499 in 18, $3,500-4,999$ in 11 , and $\geq 5,000$ in 25 patients. Mild UC was diagnosed in 25 patients and moderate UC in 44 patients. Data analysis showed there was no statistically significant difference in baseline data between the two groups, indicating that the basic demographic data of both were comparable $(\mathrm{P}>0.05)$ (see Figure 1, Table 1).

\section{Comparison of nutritional status}

The BMI score of the control group on discharge was $(19.98 \pm 2.57) \mathrm{kg} / \mathrm{m}^{2}$, and after 12 weeks this was $(21.02 \pm 3.14)$ $\mathrm{kg} / \mathrm{m}^{2}$. The BMI score of the intervention group on discharge was $(20.03 \pm 2.61) \mathrm{kg} / \mathrm{m}^{2}$, and after 12 weeks of intervention this was $(21.79 \pm 3.75) \mathrm{kg} / \mathrm{m}^{2}$. HGB was $(123.21 \pm 12.57) \mathrm{g} / \mathrm{L}$ on discharge in the control group and $(129 \pm 13.36) \mathrm{g} / \mathrm{L}$ after 12 weeks, while in the intervention group on discharge this was $(124.17 \pm 12.44) \mathrm{g} / \mathrm{L}$ on discharge and $(131 \pm 15.26) \mathrm{g} / \mathrm{L}$ after 12 weeks/L. In the control group, PA was $(225.14 \pm 30.65) \mathrm{mg} / \mathrm{L}$ on discharge and $(278.33 \pm 30.21) \mathrm{mg} / \mathrm{L}$ after 12 weeks, while in the intervention group this was $(224.27 \pm 29.25) \mathrm{mg} / \mathrm{L}$ on discharge and $(310.37 \pm 35.71) \mathrm{mg} / \mathrm{L}$ after 12 weeks of intervention. The level of ALB was $(27.95 \pm 4.98) \mathrm{g} / \mathrm{L}$ on discharge in the control group and $(33.19 \pm 5.77) \mathrm{g} / \mathrm{L}$ after 12 weeks, and in the intervention group this was $(28.06 \pm 5.22) \mathrm{g} / \mathrm{L}$ on discharge and $(36.48 \pm 6.10) \mathrm{g} / \mathrm{L}$ after 12 weeks of intervention. The TP level was $(53.26 \pm 6.12) \mathrm{g} / \mathrm{L}$ on discharge in the control group and $(59.65 \pm 7.87) \mathrm{g} / \mathrm{L}$ after 12 weeks, while in the intervention group this was $(53.17 \pm 6.25) \mathrm{g} / \mathrm{L}$ on discharge and $(65.32 \pm 8.65) \mathrm{g} / \mathrm{L}$ after 12 weeks of intervention/L. While there was no statistically significant difference in nutritional status scores between the two groups before the intervention $(\mathrm{P}>0.05)$, after 12 weeks of intervention, the PA, ALB, and TP scores of the intervention group were significantly higher than those of the control group, and the difference was statistically significant $(\mathrm{P}>0.05)$ (see Table 2$)$.

\section{Comparison of inflammatory factors between two groups}

In the control group, IL-6 the level was $(41.25 \pm 3.72) \mathrm{ng} / \mathrm{L}$ at discharge and $(32.76 \pm 2.65) \mathrm{ng} / \mathrm{L}$ after 12 weeks, and in the intervention group this was $(41.36 \pm 3.74) \mathrm{ng} / \mathrm{L}$ at discharge and $(27.6 \pm 2.13) \mathrm{ng} / \mathrm{L}$ after 12 weeks. In the control group, IL-8 was $(46.77 \pm 10.10) \mathrm{ng} / \mathrm{L}$ at discharge and $(37.2 \pm 8.63)$ $\mathrm{ng} / \mathrm{L}$ after 12 weeks, and in the intervention group, this was $(46.54 \pm 10.14) \mathrm{ng} / \mathrm{L}$ at discharge and $(32.1 \pm 7.39)$ $\mathrm{ng} / \mathrm{L}$ after 12 weeks. In the control group, TNF- $\alpha$ was $(31.9 \pm 3.61) \mathrm{ng} / \mathrm{L}$ at discharge and $(22.3 \pm 3.78) \mathrm{ng} / \mathrm{L}$ after 12 weeks, while in the intervention group, TNF- $\alpha$ was $(32.2 \pm 3.67) \mathrm{ng} / \mathrm{L}$ at discharge and $(20.9 \pm 3.31) \mathrm{ng} / \mathrm{L}$ after 12 weeks. There was no significant difference in the levels of inflammatory factors between the two groups before 


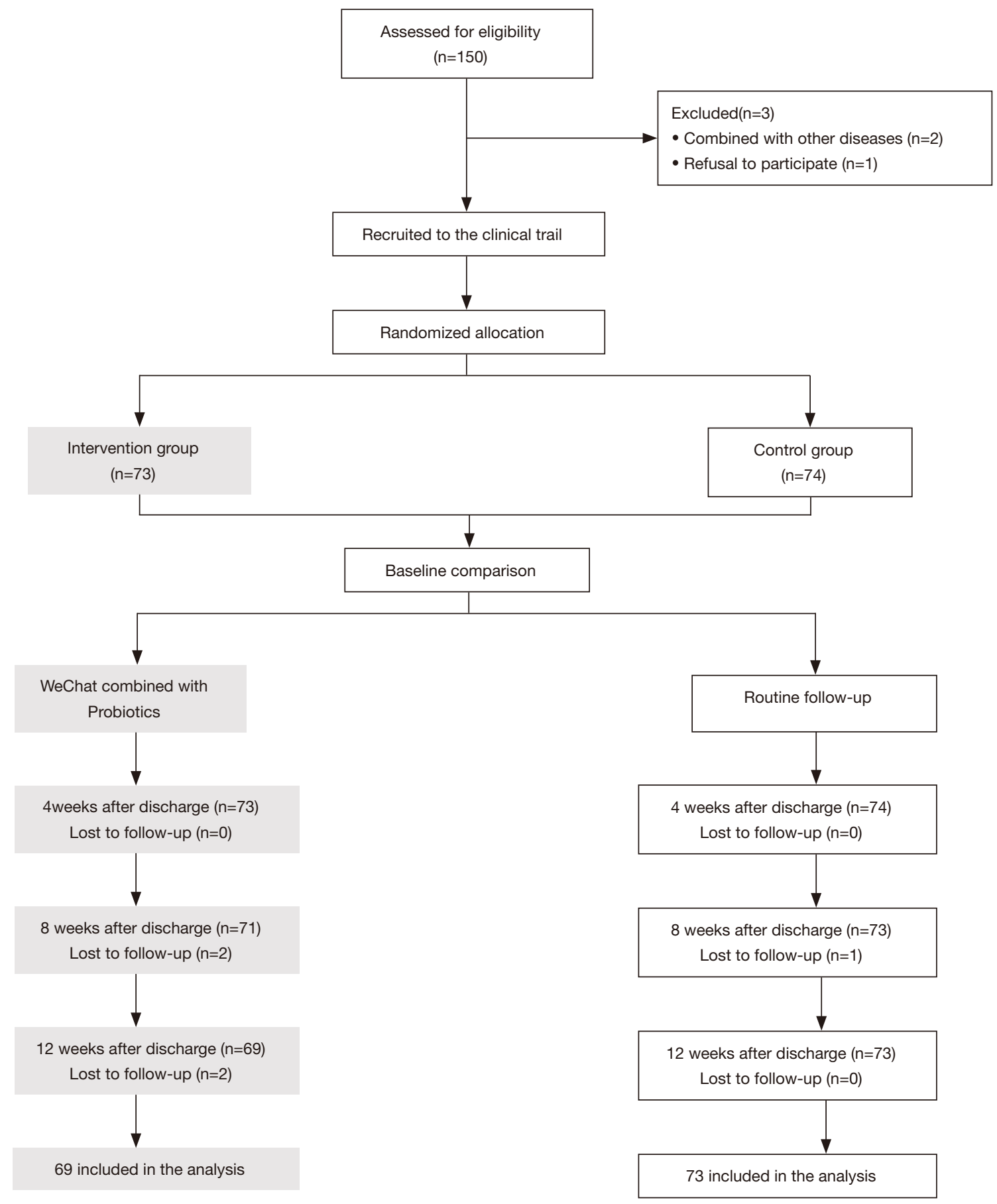

Figure 1 Flowchart for the study.

intervention $(\mathrm{P}>0.05)$. However, after 12 weeks, the scores of inflammatory factors in the intervention group were significantly higher than those in the control group $(\mathrm{P}>0.05)$ (see Table 3).

\section{Comparison of QOL between two groups}

There was no significant difference in the QOL scores between the two groups at discharge $(\mathrm{P}>0.05)$. However, after 12 weeks of intervention, the scores of both groups 
Table 1 Baseline characteristics of participants in the intervention and control group $\left(\bar{x}_{ \pm \mathrm{S}}\right) / \mathrm{N}(\%)$

\begin{tabular}{|c|c|c|c|c|c|}
\hline Variable & All $(\mathrm{N}=142), \mathrm{N}(\%)$ & $\begin{array}{l}\text { Control group }(\mathrm{N}=73) \\
\qquad \mathrm{N}(\%)\end{array}$ & $\begin{array}{l}\text { Intervention group (N=69), } \\
\qquad \mathrm{N}(\%)\end{array}$ & $t / \chi^{2}$ & $P$ \\
\hline Age (years $\pm S D)$ & $39.92 \pm 8.21$ & $39.07 \pm 7.67$ & $41.77 \pm 8.82$ & 1.139 & $0.275^{\mathrm{a}}$ \\
\hline Male & $80(56.3)$ & $40(54.8)$ & $40(58.0)$ & & \\
\hline Primary school and below & $35(24.6)$ & $20(27.4)$ & $15(21.7)$ & & \\
\hline $\begin{array}{l}\text { Secondary and higher vocational } \\
\text { education }\end{array}$ & $29(20.4)$ & $15(20.5)$ & $14(20.3)$ & & \\
\hline Junior college or above & $78(54.9)$ & $38(52.1)$ & $40(58.0)$ & & \\
\hline Married & $99(69.7)$ & $51(69.9)$ & $48(69.6)$ & & \\
\hline Monthly per capita income ( $¥$, yuan) & & & & 3.508 & $0.320^{\mathrm{b}}$ \\
\hline$<2,000$ & $41(28.9)$ & $22(30.1)$ & $19(27.5)$ & & \\
\hline $2,000-3,499$ & $39(27.5)$ & $21(28.8)$ & $18(26.1)$ & & \\
\hline $3,500-4,999$ & $28(19.7)$ & $17(23.3)$ & $11(15.9)$ & & \\
\hline$\geq 5,000$ & $34(23.9)$ & $13(17.8)$ & $21(30.4)$ & & \\
\hline Degree of disease & & & & 0.006 & $0.939^{b}$ \\
\hline
\end{tabular}

${ }^{\mathrm{a}}$, independent samples $t$-test; ${ }^{\mathrm{b}}$, Chi-square test.

Table 2 Comparison of nutritional status between two groups before and after intervention $\left(\bar{x}_{ \pm \mathrm{s}}\right)$

\begin{tabular}{|c|c|c|c|c|}
\hline Variable & \multicolumn{2}{|c|}{ Before intervention } & \multicolumn{2}{|c|}{ After intervention } \\
\hline BMI $\left(\mathrm{kg} / \mathrm{m}^{2}\right)$ & $19.98 \pm 2.57$ & $20.03 \pm 2.61$ & $21.02 \pm 3.14$ & $21.79 \pm 3.75$ \\
\hline HGB (g/L) & $123.21 \pm 12.57$ & $124.17 \pm 12.44$ & $129 \pm 13.36$ & $131 \pm 15.26$ \\
\hline $\mathrm{PA}(\mathrm{mg} / \mathrm{L})$ & $225.14 \pm 30.65$ & $224.27 \pm 29.25$ & $278.33 \pm 30.21^{a}$ & $310.37 \pm 35.71^{\mathrm{ab}}$ \\
\hline TP (g/L) & $53.26 \pm 6.12$ & $53.17 \pm 6.25$ & $59.65 \pm 7.87^{\mathrm{a}}$ & $65.32 \pm 8.65^{\mathrm{ab}}$ \\
\hline
\end{tabular}

Compared with before treatment, ${ }^{\mathrm{a}} \mathrm{P}<0.05$; compared with the control group, ${ }^{\mathrm{b}} \mathrm{P}<0.05$. 
Table 3 Comparison of inflammatory factors between two groups before and after intervention $\left(\bar{x}_{ \pm \mathrm{s}}\right)$

\begin{tabular}{|c|c|c|c|c|}
\hline Variable & \multicolumn{2}{|c|}{ Before intervention } & \multicolumn{2}{|c|}{ After intervention } \\
\hline IL-6 (ng/L) & $41.25 \pm 3.72$ & $41.36 \pm 3.74$ & $32.76 \pm 2.65^{a}$ & $27.6 \pm 2.13^{\mathrm{ab}}$ \\
\hline IL-8 (ng/L) & $46.77 \pm 10.10$ & $46.54 \pm 10.14$ & $37.2 \pm 8.63^{\mathrm{a}}$ & $32.1 \pm 7.39^{\mathrm{ab}}$ \\
\hline TNF- $\alpha$ (ng/L) & $31.9 \pm 3.61$ & $32.2 \pm 3.67$ & $22.3 \pm 3.78^{\mathrm{a}}$ & $20.9 \pm 3.31^{\mathrm{ab}}$ \\
\hline
\end{tabular}

Compared with before treatment, ${ }^{\mathrm{a}} \mathrm{P}<0.05$; compared with the control group, ${ }^{\mathrm{b}} \mathrm{P}<0.05$.

Table 4 Comparison of SF-36 score between two groups before and after intervention $(\bar{x} \pm \mathrm{S})$

\begin{tabular}{lcccc}
\hline \multirow{2}{*}{ Variable } & \multicolumn{2}{c}{ Before intervention } & \multicolumn{2}{c}{ After intervention } \\
\cline { 2 - 5 } PF & Control group & Intervention group & Control group & Intervention group \\
nyP & $52.17 \pm 4.32$ & $52.08 \pm 4.51$ & $63.04 \pm 5.76^{\mathrm{a}}$ & $77.02 \pm 7.34^{\mathrm{ab}}$ \\
BP & $59.12 \pm 5.71$ & $59.34 \pm 5.68$ & $64.35 \pm 4.23$ & $76.56 \pm 4.87^{\mathrm{ab}}$ \\
GH & $64.56 \pm 4.45$ & $65.17 \pm 4.53$ & $77.36 \pm 7.19^{\mathrm{a}}$ & $79.54 \pm 7.89^{\mathrm{a}}$ \\
VT & $51.34 \pm 5.07$ & $50.96 \pm 5.31$ & $71.34 \pm 6.77^{\mathrm{a}}$ & $75.39 \pm 5.34^{\mathrm{a}}$ \\
SF & $53.76 \pm 4.09$ & $53.31 \pm 4.42$ & $65.79 \pm 4.31^{\mathrm{a}}$ & $66.86 \pm 4.23^{\mathrm{a}}$ \\
RE & $55.71 \pm 4.56$ & $55.46 \pm 4.73$ & $68.24 \pm 4.67^{\mathrm{a}}$ & $78.49 \pm 8.07^{\mathrm{ab}}$ \\
MH & $63.45 \pm 5.59$ & $64.01 \pm 5.67$ & $69.54 \pm 5.35^{\mathrm{a}}$ & $72.39 \pm 6.32^{\mathrm{a}}$ \\
\hline
\end{tabular}

Compared with before treatment, ${ }^{\mathrm{a}} \mathrm{P}<0.05$; compared with the control group, ${ }^{\mathrm{b}} \mathrm{P}<0.05$.

Table 5 Comparison of IBDQ scores of two groups before and after intervention $\left(\bar{x}_{ \pm s}\right)$

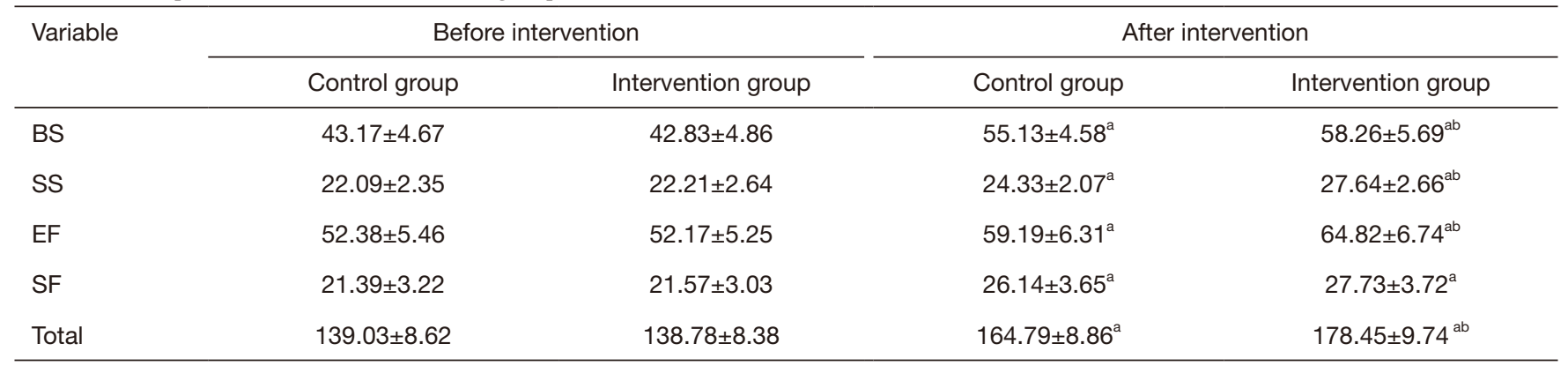

Compared with before treatment, ${ }^{\mathrm{a}} \mathrm{P}<0.05$; compared with the control group, ${ }^{\mathrm{b}} \mathrm{P}<0.05$.

were higher than before treatment, and the scores of BS, $\mathrm{SS}$, and EF in sf-36pf, RP, SF, and IBQD in the intervention group were significantly higher than those in the control group $(\mathrm{P}<0.05)$ (see Tables 4 and 5$)$.

\section{Discussion}

$\mathrm{UC}$ is a chronic disease, UC patients with long disease duration have an increased risk of developing colorectal cancer (CRC) (10). At present, it is believed that the canceration pattern of UC follows the process of "inflammation, low-grade intraepithelial neoplasia, highgrade intraepithelial neoplasia, canceration" (11). The specific mechanism of UC carcinogenesis is still unclear. At present, it is believed that the tumorigenesis caused by chronic inflammation is mainly related to various 
inflammatory factors and inflammatory related pathways. Inflammatory factors, such as TNF- $\alpha$, IL-6, Nuclear factor$\kappa \mathrm{B}(\mathrm{NF}-\kappa \mathrm{B})$, can promote the inflammatory response of intestinal epithelial cells, promote the dysplasia of epithelial cells, and promote the occurrence and development of tumor, but the specific mechanism and related signaling pathways are not clear. The clinical treatment strategy of $\mathrm{UC}$ is to induce and maintain the remission of inflammation, and to avoid the occurrence of colectomy and colorectal cancer. At present, first-line treatment drugs mainly include aminosalicylates, corticosteroids, biological drugs, and immunosuppressants (12). However, not all $\mathrm{UC}$ patients benefit from these treatments, and there is a considerable risk of side effects. Therefore, greater attention has been paid to treatments such as health intervention, stem cell therapy, traditional Chinese medicine, and microecological agents. Under normal circumstances, a large number of intestinal flora inhabit the human gut where they play a defensive role by assisting the nutrient digestion, absorption, and secretion of bacteriostatic components, promote the regeneration of intestinal blood vessels and intestinal epithelium, and maintain the best balance of intestinal immunity. Studies have shown that the composition and spatial distribution of UC patients differs from that of healthy people, and it is generally accepted that intestinal flora play an important role in the occurrence and development of UC. This provides a theoretical basis for the use of probiotics treatment or auxiliary treatment of the disease. Traditional treatment drugs such as salicylic acid, glucocorticoids, and immunosuppressors focus on controlling active inflammation and regulating immune disorders, but the low remission rate and high recurrence and adverse reaction rate complicate this treatment. The typical characteristic of UC is intestinal microecological disorder. Therefore, more and more attention has been paid to the treatment strategy of using probiotics to repair damaged intestinal microbiota to alleviate IBD (13). Probiotics such as Ifidobacterium longum, Lactobacillus, and E. coli nissle 1917 can reduce the levels of proinflammatory cytokines in the intestine of experimental colitis animals, alleviate damage to colon tissue, and repair the balance of intestinal microecology (14-16). A metaanalysis showed that VSL 3 (Streptoccus thermophilus, Lactobacillus acidophilus, Lactobacillus delbrueckii ssp. bulgaricus, Lactobacillus paracasei, Lactobacillus plantarum, Bifidobacterium longum, Bifidobacterium infantis, and Bifidobacterium breve) can improve the performance of the composite bacteria remission rate of UC patients and effectively prevent recurrence $(17,18)$. Researchers believe that probiotics can provide an alternative or auxiliary method for traditional treatment by changing the intestinal flora and regulating the host immune system. In vitro and animal studies have shown that probiotics can affect the level of inflammatory cytokines, improve the production of some mediators involved in intestinal inflammatory responses, and enhance intestinal permeability $(19,20)$. Probiotics combined with mesalazine can relieve the clinical symptoms and reduce the level of inflammatory factors in patients with mild to moderate active UC (21). Bifid triple viable capsules are composed of three probiotic mixtures (22), Bifidobacterium, Enterococcus, and Lactobacillus acidophilus. Due to their advanced coating technology, the drug effect reaches the intestinal tract, crosses the gastric acid barrier, improves the endogenous defense barrier, and eliminates pathogenic bacteria in the intestine. This enhances the intestinal flora environment, achieving the treatment effect.

At the same time, long-term continuous and frequent monitoring is very important in the management of chronic disease, and with the development of information technology and e-health, the nature of medical consultations has changed (23). The widespread use of smartphones in China has seen the introduction of mobile health applications which have the potential to greatly improve chronic disease management. WeChat is a very popular social application that is easy to operate and can provide a variety of functions, such as text and voice messages, free voice and video calls, group chat, and subscription to public accounts and applets. Therefore, based on the applications of WeChat in UC health management and the characteristics of WeChat technology, we constructed a UC WeChat management platform to study its value in UC management.

The main indicators of nutritional status are BMI, HGB, PA, ALB, and TP. The half-life of PA is short, and once the body lacks heat or loses protein, its level is rapidly reduced, making it highly sensitive to the assessment of human malnutrition, and an important reference index of nutritional status. The results of the current study showed that serum ALB, PA, and TP levels in the intervention group were significantly higher than those in the control group $(\mathrm{P}<0.05)$.

TNF- $\alpha$ is mainly produced by activated monocytes and macrophages and is an important inflammatory mediator. TNF- $\alpha$ can increase vascular permeability, improve the response of target cells to a variety of cytokines, and 
ultimately release a variety of inflammatory factors to promote inflammatory response. IL-6 is mainly secreted by activated macrophages, lymphocytes, and epithelial cells. The role of IL-6 in the acute inflammatory response is mainly manifested in its pro-inflammatory effect on a variety of cells and the induction of acute reactive protein production in liver tissue. Therefore, the serum IL-6 level in patients with active $\mathrm{UC}$ is significantly higher than that in healthy adults and patients in remission. IL- 8 is a powerful chemokine and activator of neutrophils, and is produced by monocytes, epithelial cells, epidermal cells, fibroblasts, and T lymphocytes stimulated by IL-1, TNF, and lipopolysaccharide (LPS). Its biological function is to activate neutrophils, promote the lysosomal enzyme activity and phagocytosis of neutrophils, and to evoke a chemotactic effect on basophils and T cells. At present, it is believed that the inflammatory response induced by TNF and IL-6 is mediated to a large extent by promoting the production of chemokines represented by IL-8. After treatment, the levels of TNF- $\alpha$, IL- 6 , and IL- 8 in the observation group were significantly lower than those in the control group $(\mathrm{P}<0.05)$. This showed that the two groups of treatment could effectively enhance the anti-inflammatory effect and reduce the inflammatory reaction, and the effect of the intervention group was more significant than that of the control group.

QOL is the subjective experience of individuals in different cultures and value systems about the living conditions related to their life goals, wishes, standards, and things they care about. It includes four aspects; individual physiology, social function, material state, and psychology. QOL mainly refers to the subjective evaluation of the individual, which is rooted in the cultural and social environment (24), and is suitable for all individuals and groups. The concept of health-related quality of life (HRQOL) is commonly used in medical research, and although HRQoL and QOL have different concepts, their specific research contents are consistent $(25,26)$. In the present study, the 12-week QOL scores in the two groups increased, and the increase in the intervention group was more significant. In conclusion, our research shows that probiotics combined with a WeChat platform intervention can significantly improve the nutritional status of patients, reduce the level of inflammatory factors, and improve the QOL in patients with UC.

However, the results should be approached with caution due to the small number of participants and unreliable methods used in the trial, and more robust studies with more participants are required to confirm the results.

\section{Acknowledgments}

Funding: None.

\section{Footnote}

Reporting Checklist: The authors have completed the CONSORT reporting checklist. Available at http://dx.doi. org/apm-21-1056

Trial Protocol: Available at http://dx.doi.org/apm-21-1056

Data Sharing Statement: Available at http://dx.doi.org/apm21-1056

Conflicts of Interest: All authors have completed the ICMJE uniform disclosure form (available at http://dx.doi.org/ apm-21-1056). The authors have no conflicts of interest to declare.

Ethical Statement: The authors are accountable for all aspects of the work in ensuring that questions related to the accuracy or integrity of any part of the work are appropriately investigated and resolved. This study was conducted in accordance with the ethical principles of the Declaration of Helsinki (as revised in 2013) and has been approved by the ethics committee of the Affiliated Hospital of Jiangnan University (No. 201804367). Informed consent was taken from all the patients.

Open Access Statement: This is an Open Access article distributed in accordance with the Creative Commons Attribution-NonCommercial-NoDerivs 4.0 International License (CC BY-NC-ND 4.0), which permits the noncommercial replication and distribution of the article with the strict proviso that no changes or edits are made and the original work is properly cited (including links to both the formal publication through the relevant DOI and the license). See: https://creativecommons.org/licenses/by-nc-nd/4.0/.

\section{References}

1. Kucharzik T, Koletzko S, Kannengiesser K, et al. Ulcerative Colitis-Diagnostic and Therapeutic Algorithms. Dtsch Arztebl Int 2020;117:564-74.

2. Wei SC, Sollano J, Hui YT, et al. Epidemiology, burden of disease, and unmet needs in the treatment of ulcerative colitis in Asia. Expert Rev Gastroenterol Hepatol 
2021;15:275-89.

3. He Q, Li Jd. Research progress in the epidemiology of inflammatory bowel disease. J Practical Med 2019;35:2962-6.

4. Hindryckx P, Jairath V, D'Haens G. Acute severe ulcerative colitis: from pathophysiology to clinical management. Nat Rev Gastroenterol Hepatol 2016;13:654-64.

5. Molodecky NA, Soon IS, Rabi DM, et al. Increasing incidence and prevalence of the inflammatory bowel diseases with time, based on systematic review. Gastroenterology 2012;142:46-54.e42; quiz e30.

6. Zhang QL, Xu N, Huang ST, et al. WeChat-Assisted Preoperative Health Education Reduces Burden of Care on Parents of Children with Simple Congenital Heart Disease: a Prospective Randomized Controlled Study. Braz J Cardiovasc Surg 2020. [Epub ahead of print].

7. Peng Z, Li L, Chen Y, et al. WeChat app-based reinforced education improves the quality of opioid titration treatment of cancer-related pain in outpatients: a randomized control study. BMC Cancer 2020;20:852.

8. Song Y, Xie X, Chen Y, et al. The effects of WeChatbased educational intervention in patients with ankylosing spondylitis: a randomized controlled trail. Arthritis Res Ther 2021;23:72.

9. Brown AC, Rampertab SD, Mullin GE. Existing dietary guidelines for Crohn's disease and ulcerative colitis. Expert Rev Gastroenterol Hepatol 2011;5:411-25.

10. Yang L, Levi E, Du JH, et al. Associations between markers of colorectal cancer stem cells, mutation, microRNA and the clinical features of ulcerative colitis. Colorectal Dis 2016;18:0185-93.

11. Prideaux L, Kamm MA, De Cruz PP, et al. Inflammatory bowel disease in Asia: a systematic review. J Gastroenterol Hepatol 2012;27:1266-80.

12. Ungaro R, Mehandru S, Allen PB, et al. Ulcerative colitis. Lancet 2017;389:1756-70.

13. Ganji-Arjenaki M, Rafieian-Kopaei M. Probiotics are a good choice in remission of inflammatory bowel diseases: A meta analysis and systematic review. J Cell Physiol 2018;233:2091-103.

14. Srutkova D, Schwarzer M, Hudcovic T, et al. Bifidobacterium longum CCM 7952 Promotes Epithelial Barrier Function and Prevents Acute DSS-Induced Colitis in Strictly Strain-Specific Manner. PLoS One 2015;10:e0134050.

15. Rodríguez-Nogales A, Algieri F, Garrido-Mesa J, et al. Differential intestinal anti-inflammatory effects of
Lactobacillus fermentum and Lactobacillus salivarius in DSS mouse colitis: impact on microRNAs expression and microbiota composition. Mol Nutr Food Res 2017;61(11). doi: 10.1002/mnfr.201700144.

16. Rodríguez-Nogales A, Algieri F, Garrido-Mesa J, et al. The Administration of Escherichia coli Nissle 1917 Ameliorates Development of DSS-Induced Colitis in Mice. Front Pharmacol 2018;9:468.

17. Shen J, Zuo ZX, Mao AP. Effect of probiotics on inducing remission and maintaining therapy in ulcerative colitis, Crohn's disease, and pouchitis: meta-analysis of randomized controlled trials. Inflamm Bowel Dis 2014;20:21-35.

18. Peng L, Zhong Y, Wang A, et al. Probiotics combined with aminosalicylic acid affiliates remission of ulcerative colitis: a meta-analysis of randomized controlled trial. Biosci Rep 2019;39:BSR20180943.

19. Tursi A, Brandimarte G, Papa A, et al. Treatment of relapsing mild-to-moderate ulcerative colitis with the probiotic VSL\#3 as adjunctive to a standard pharmaceutical treatment: a double-blind, randomized, placebo-controlled study. Am J Gastroenterol 2010;105:2218-27.

20. Macho Fernandez E, Valenti V, Rockel C, et al. Antiinflammatory capacity of selected lactobacilli in experimental colitis is driven by NOD2-mediated recognition of a specific peptidoglycan-derived muropeptide. Gut 2011;60:1050-9.

21. Peran L, Sierra S, Comalada M, et al. A comparative study of the preventative effects exerted by two probiotics, Lactobacillus reuteri and Lactobacillus fermentum, in the trinitrobenzenesulfonic acid model of rat colitis. Br J Nutr 2007;97:96-103.

22. Qiu Cl, Yan H, Wu Xj, et al. Study on the microecological changes of ulcerative colitis and the therapeutic effect of bifidobacteria. Chinese General Practice 2014;17:3077-82.

23. Panahiazar M, Taslimitehrani V, Jadhav A, et al. Empowering Personalized Medicine with Big Data and Semantic Web Technology: Promises, Challenges, and Use Cases. Proc IEEE Int Conf Big Data 2014;2014:790-5.

24. The Development of the World Health Organization Quality of Life Assessment Instrument (the WHOQOL). Quality of Life Assessment: International Perspectives; 1994 1994//; Berlin, Heidelberg: Springer Berlin Heidelberg.

25. Xu F, Dahlhamer JM, Zammitti EP, et al. Health-Risk Behaviors and Chronic Conditions Among Adults with Inflammatory Bowel Disease - United States, 2015 and 
2016. MMWR Morb Mortal Wkly Rep 2018;67:190-5.

26. Iglesias-Rey M, Barreiro-de Acosta M, Caamaño-Isorna F, et al. Psychological Factors Are Associated with Changes in the Health-related Quality of Life in Inflammatory

Cite this article as: Ou Q, Wang L, Wang K, Shao P. Effect of probiotics supplementation combined with WeChat platform health management on nutritional status, inflammatory factors, and quality of life in patients with mild-to-moderate ulcerative colitis: a randomized trial. Ann Palliat Med 2021;10(6):66066616. doi: 10.21037/apm-21-1056
Bowel Disease. Inflamm Bowel Dis 2014;20:92-102.

(English Language Editor: B. Draper) 